Federal Reserve Bank of Minneapolis

Research Department Staff Report 243

January 1998

\title{
Dynamic Equilibrium Economies: A Framework for Comparing Models and Data
}

\author{
Francis X. Diebold* \\ University of Pennsylvania \\ and National Bureau of Economic Research \\ Lee E. Ohanian* \\ University of Minnesota \\ and Federal Reserve Bank of Minneapolis \\ Jeremy Berkowitz* \\ Federal Reserve Board
}

\begin{abstract}
We propose a constructive, multivariate framework for assessing agreement between (generally misspecified) dynamic equilibrium models and data, a framework which enables a complete second-order comparison of the dynamic properties of models and data. We use bootstrap algorithms to evaluate the significance of deviations between models and data, and we use goodness-of-fit criteria to produce estimators that optimize economically relevant loss functions. We provide a detailed illustrative application to modeling the U.S. cattle cycle.
\end{abstract}

*The co-editor and referees provided helpful and constructive input, as did participants at meetings of the Econometric Society, ECARE/CEPR, NBER, and numerous university seminars. José Lopez provided dedicated research assistance in the early stages of this project. We gratefully acknowledge helpful discussion from Bill Brown, Fabio Canova, Tim Cogley, Bob Lucas, Ellen McGrattan, Danny Quah, Lucrezia Reichlin, Sherwin Rosen, Chris Sims, Tony Smith, Jim Stock, Mark Watson, and especially Lars Hansen, Adrian Pagan, Tom Sargent, and Mike Wickens . All remaining errors and inaccuracies are ours. We thank the National Science Foundation, the Sloan Foundation, and the University of Pennsylvania Research Foundation for support. The views expres sed herein are those of the authors and not necessarily those of the Federal Reserve Bank of Minneapolis or the Federal Reserve System. 


\section{Introduction}

Dynamic equilibrium models are now used routinely in many fields. Such models, for example, have been used to address a variety of macroeconomic issues, including business cycle fluctuations, economic growth, and the effects of government policies. ${ }^{1}$ Additional prominent fields of application include international economics, public economics, industrial organization, labor economics, and agricultural economics. ${ }^{2}$

At present, however, many important questions regarding the empirical implementation of dynamic equilibrium models remain incompletely answered. The questions fall roughly into two methodological groups. One group involves issues related to assessing model adequacy, and the other involves issues related to model estimation. We contribute to an emerging literature that has begun to deal with both issues, including King and Watson (1992, 1996), Watson (1993), Canova, Finn, and Pagan (1994), Kim and Pagan (1994), Leeper and Sims (1994), Pagan (1994), Cogley and Nason (1995), and Hansen, McGrattan, and Sargent (1997). A 1996 Journal of Economic Perspectives symposium (Hansen and Heckman, 1996; Kydland and Prescott, 1996; Sims, 1996) focuses on these issues, and two important messages emerge: (1) Dynamic equilibrium models, like all models, are intentionally simple abstractions and therefore should not be construed as the true data generating process.

${ }^{1}$ Among many others, see Kydland and Prescott (1982), Hansen (1985), Christiano and Eichenbaum (1995), and Rotemberg and Woodford (1996, 1997) (business cycle fluctuations); Lucas (1988), Jones and Manuelli (1990), Rebelo (1991), and Greenwood, Hercowitz, and Krusell (1997) (economic growth); and Lucas (1990), Cooley and Hansen (1992), and Ohanian (1997) (effects of government policies).

2 Among many others, see Backus, Kehoe, and Kydland (1994) (international economics); Auerbach and Kotlikoff (1987) (public economics); Ericson and Pakes (1995) (industrial organization); Rust (1989) (labor economics); and Rosen, Murphy, and Scheinkman (1994) (agricultural economics). 
Formal methods should be developed and used to help us assess the models more thoroughly. In this paper, we take a step toward meeting those goals.

Some parts of our framework are new, while others build on earlier work in interesting ways. In many respects, our work begins where Watson (1993) ends. With an eye toward future research, Watson notes that "one of the most informative diagnostics ... is the plot of the model and data spectra," and he recommends that in the future, researchers "present both model and data spectra as a convenient way of comparing their complete set of second moments." Our methods are based directly on comparison of model and data spectra. They can be used to assess the performance of a model (for a given set of parameters), to estimate model parameters, and to test hypotheses about parameters or models. To elaborate, our approach is

A. Frequency-domain and multivariate. Working in the frequency domain enables decomposition of variation across frequencies, which is often useful, and the multivariate focus facilitates simple examination of cross-variable correlations and lead-lag relationships at the frequencies of interest.

B. Based on a full second-order comparison of model and data dynamics, in contrast to a common approach used in the business cycle literature, in which only a few variances and covariances from the model economy and the actual economy are compared. The spectrum provides a complete summary of Gaussian time series dynamics and an approximate summary of non-Gaussian time series dynamics.

C. Based on the realistic assumption that all models are misspecified. We regard all of the models we entertain as false, in which case traditional statistical methods lose some of their appeal.

D. Graphical and constructive. The framework permits us to assess visually and quickly the dimensions along which a model performs well, and the dimensions along which it performs poorly.

E. Based on a common set of tools that can be used by researchers with potentially very different objectives and research strategies. The framework can be used to 
evaluate strictly calibrated models, and it can also be used formally to estimate and test models.

F. Designed to facilitate statistical inference about objects estimated from data, including spectra, goodness-of-fit measures, model parameters, and test statistics. Bootstrap methods play an important role in that regard. We develop and use a simple nonparametric bootstrap algorithm.

G. Mathematically convenient. Under regularity conditions, the spectrum is a bounded continuous function, which makes for convenient mathematical developments.

All of the classical ideas of business cycle analysis discussed, for example, by Lucas (1977) have spectral analogs, ranging from univariate persistence (typical spectral shape) to multivariate issues of comovement (coherence) and lead-lag relationships (phase shifts) at business cycle frequencies. We highlight these links and draw upon the business cycle literature for motivation in the methodological Sections 2 and 3. The methods we develop, however, are not wed to macroeconomics in any way; rather, they can be used in a variety of fields. Therefore, to maintain balance and to introduce researchers in different areas to the use of our framework, we apply our methods to a simple and accessible, yet rich, microeconomic model in Section 4. We conclude in Section 5.

\section{Assessing Agreement Between Model and Data}

Our basic strategy is to assess models by comparing model spectra to data spectra. Our goal is provision of a graphical framework that facilitates visual comparisons of model spectra to interval estimates of data spectra. We compute model spectra exactly (either analytically or numerically); thus, the spectra have no sampling uncertainty. Sampling error does, however, affect the sample data spectra, which are of course just estimates of true but unknown (population) data spectra. We exploit well-established procedures for estimating 
spectra, and we develop and use bootstrap techniques to assess the sampling uncertainty of estimated spectra. ${ }^{3}$

$\underline{\text { Estimating Spectra }}$

Consider the N-variate linearly regular covariance stationary stochastic process,

$$
\begin{gathered}
\mathrm{y}_{\mathrm{t}}=\mu+\mathrm{B}(\mathrm{L}) \varepsilon_{\mathrm{t}}=\mu+\sum_{\mathrm{i}=-\infty}^{\infty} \mathrm{B}_{\mathrm{i}} \varepsilon_{\mathrm{t}-\mathrm{i}} \\
\mathrm{E}\left(\varepsilon_{\mathrm{t}} \varepsilon_{\mathrm{s}}^{\prime}\right)= \begin{cases}\Omega & \text { if } \mathrm{t}=\mathrm{s} \\
0 & \text { otherwise }\end{cases}
\end{gathered}
$$

where $\mathrm{E}\left(\varepsilon_{\mathrm{t}}\right)=0, \mathrm{~B}_{0}=\mathrm{I}$, and the coefficients are square summable (in the matrix sense).

The autocovariance function is $\Gamma(\tau)=\sum_{i=-\infty}^{\infty} B_{i} \Omega B_{i+\tau}^{\prime}$, and the spectral density function is $\mathrm{F}(\omega)=\frac{1}{2 \pi} \sum_{\tau=-\infty}^{\infty} \Gamma(\tau) \mathrm{e}^{-\mathrm{i} \omega \tau},-\pi<\omega<\pi$.

Consider now a generic off-diagonal element of $\mathrm{F}(\omega), \mathrm{f}_{\mathrm{kl}}(\omega)$. In polar form, the crossspectral density is $\mathrm{f}_{\mathrm{kl}}(\omega)=\operatorname{ga}_{\mathrm{kl}}(\omega) \exp \left[\mathrm{i} \operatorname{ph}_{\mathrm{kl}}(\omega)\right]$, where $\mathrm{ga}_{\mathrm{kl}}(\omega)=\left[\mathrm{re}^{2}\left(\mathrm{f}_{\mathrm{kl}}(\omega)\right)+\right.$ $\left.\operatorname{im}^{2}\left(f_{\mathrm{kl}}(\omega)\right)\right]^{1 / 2}$ is the gain or amplitude and where $\mathrm{ph}_{\mathrm{kl}}(\omega)=\arctan \left\{\mathrm{im}\left(\mathrm{f}_{\mathrm{kl}}(\omega)\right) / \operatorname{re}\left(\mathrm{f}_{\mathrm{kl}}(\omega)\right)\right\}$ is the phase. As is well known, the gain tells how the amplitude of $y_{1}$ is multiplied in contributing to the amplitude of $\mathrm{y}_{\mathrm{k}}$ at frequency $\omega$, and phase measures the lead of $\mathrm{y}_{\mathrm{k}}$ over $\mathrm{y}_{1}$ at frequency $\omega$. (The phase shift in time units is $\mathrm{ph}(\omega) / \omega$.) We shall often find it convenient to examine coherence rather than gain, where the coherence is defined as

${ }^{3}$ Alternatively, we could fix the data spectrum and assess the sampling error in the model spectrum by simulating repeated realizations from the model. The two approaches are essentially complementary, roughly analogous to the Wald and Lagrange multiplier testing perspectives. See, for example, Gregory and Smith (1991). 
$\operatorname{coh}_{\mathrm{kl}}(\omega)=\frac{\mathrm{ga}_{\mathrm{kl}}^{2}(\omega)}{\mathrm{f}_{\mathrm{kk}}(\omega) \mathrm{f}_{\mathrm{ll}}(\omega)}$, which measures the squared correlation between $\mathrm{y}_{\mathrm{k}}$ and $\mathrm{y}_{1}$ at frequency $\omega$.

Given a sample path $\left\{\mathrm{y}_{1 \mathrm{t}}, \ldots, \mathrm{y}_{\mathrm{Nt}}\right\}_{\mathrm{t}=1}^{\mathrm{T}}$, we estimate the $\mathrm{N} \times 1$ mean vector $\mu$ with $\overline{\mathrm{y}}=\left(\overline{\mathrm{y}}_{1}, \ldots, \overline{\mathrm{y}}_{\mathrm{N}}\right)^{\prime}$. From this point onward, we assume that the sample means have been subtracted from the variables. We estimate the autocovariance function with $\hat{\Gamma}(\tau)=\left[\hat{\gamma}_{\mathrm{kl}}(\tau)\right]$ $(\mathrm{k}=1, \ldots, \mathrm{N}, \mathrm{l}=1, \ldots, \mathrm{N})$, where $\hat{\gamma}_{\mathrm{kl}}(\tau)=\frac{1}{\mathrm{~T}} \sum_{\tau=1}^{\mathrm{T}-|\tau|} \mathrm{y}_{\mathrm{kt}} \mathrm{y}_{1, \mathrm{t}+\tau}, \tau=0, \pm 1, \ldots, \pm(\mathrm{T}-1)$. We estimate the spectral density matrix using the Blackman-Tukey lag-window approach in which we replace the sample spectral density function, $\hat{\mathrm{F}}\left(\omega_{\mathrm{j}}\right)=\frac{1}{2 \pi} \sum_{\tau=-(\mathrm{T}-1)}^{(\mathrm{T}-1)} \hat{\Gamma}(\tau) \mathrm{e}^{-\mathrm{i} \omega_{\mathrm{j}} \tau}$ $\left(\omega_{\mathrm{j}}=\frac{2 \pi \mathrm{j}}{\mathrm{T}}, \mathrm{j}=1, \ldots, \frac{\mathrm{T}}{2}-1\right)$, with one involving the windowed sample autocovariance sequence, $F^{*}\left(\omega_{\mathrm{j}}\right)=\frac{1}{2 \pi} \sum_{\tau=-(\mathrm{T}-1)}^{(\mathrm{T}-1)} \Lambda(\tau) \hat{\Gamma}(\tau) \mathrm{e}^{-\mathrm{i} \omega \tau}$, where $\Lambda(\tau)$ is a matrix of lag windows. The Blackman-Tukey procedure results in a consistent estimator if we adjust the lag window $\Lambda(\tau)$ with sample size in such a way that variance and bias decline simultaneously. We then obtain the sample coherence and phase at any frequency $\omega_{\mathrm{j}}$ by transforming the appropriate elements of $F^{*}\left(\omega_{j}\right)$.

\section{$\underline{\text { Assessing Sampling Variability }}$}

A key issue for our purposes is how to ascertain the sampling variability of the estimated spectral density function. To do so, we use an algorithm for resampling from time series data, an algorithm which we call the Cholesky factor bootstrap and which is closely related to the Ramos (1988) bootstrap. The main difference is that Ramos proceeds in the frequency domain, whereas we develop the Cholesky factor bootstrap in the time domain. First we compute the Cholesky factor of the sample covariance matrix of the series of interest. We then exploit the fact that up to the second order, the series of interest can be written as the 
product of the Cholesky factor and serially uncorrelated disturbances, which can be easily bootstrapped by using parametric or nonparametric procedures. ${ }^{4}$

First we need some definitions and notation. Let $\mathrm{z}_{\mathrm{t}}=\left(\mathrm{y}_{1 \mathrm{t}}, \ldots, \mathrm{y}_{\mathrm{Nt}}\right)^{\prime}$, and let $\mathrm{z}=\left(\mathrm{z}_{1}^{\prime}, \mathrm{z}_{2}^{\prime}, \ldots, \mathrm{z}_{\mathrm{T}}^{\prime}\right)^{\prime}$. Then $\mathrm{z} \sim(1 \otimes \mu, \Sigma)$, where 1 is an $\mathrm{N}$-dimensional column vector of ones and $\Sigma=\operatorname{Toeplitz}(\Gamma(0), \Gamma(1), \ldots, \Gamma(\mathrm{T}-1))$. By symmetry and positive definiteness, we can write $\Sigma=\mathrm{PP}^{\prime}$, where the unique Cholesky factor $\mathrm{P}$ is lower triangular. We estimate $\Sigma$ by $\hat{\Sigma}=\operatorname{Toeplitz}(\hat{\Gamma}(0), \hat{\Gamma}(1), \ldots, \hat{\Gamma}(\mathrm{T}-1))$, where $\hat{\Gamma}(\tau)=\frac{1}{\mathrm{~T}} \sum_{\mathrm{t}=1}^{\mathrm{T}-|\tau|} \mathrm{z}_{\mathrm{t}} \mathrm{z}_{\mathrm{t}+|\tau|}^{\prime}$, $\tau=0, \pm 1, \ldots, \pm(\mathrm{T}-1)$. This ensures that we can write $\hat{\Sigma}=\hat{\mathrm{P}} \hat{\mathrm{P}}^{\prime}$, where the unique Cholesky factor $\hat{\mathrm{P}}$ is lower triangular. Now let $\left\{\lambda_{|\mathrm{i}-\mathrm{j}|}\right\}_{|\mathrm{i}-\mathrm{j}|=0}^{\mathrm{T}-1}$ be a set of decreasing weights applied to the successive off-diagonal blocks of $\hat{\Sigma}$, and call the resulting matrix $\Sigma^{*}$. Finally, let $\mathrm{P}^{*}$ be the Cholesky factor of $\Sigma^{*}$.

The fact that $\mathrm{z} \sim\left(1 \otimes \mu, \mathrm{PP}^{\prime}\right)$ implies that data generated by drawing $\varepsilon^{(\mathrm{i})} \underset{\sim}{\text { iid }}\left(0, \mathrm{I}_{\mathrm{NT}}\right)$ and forming

$$
\mathrm{Z}^{(\mathrm{i})}=\mu_{\mathrm{z}}+\mathrm{P} \varepsilon^{(\mathrm{i})}
$$

where $\mu_{z}=1 \otimes \mu$, will have the same second-order properties as the observed data. In practice, we replace the unknown population first and second moments with the consistent

${ }^{4}$ Note that the Cholesky factor bootstrap will miss nonlinear dynamics such as GARCH. It is designed to capture only second-order dynamics, in identical fashion to standard (as opposed to higher-order) spectral analysis. Users should be cautious in employing our procedures if nonlinearities are suspected to be operative, as may be the case with high-frequency financial data, or with models of prices in which inventory constraints may be important, as in Deaton and Laroque (1995). Such nonlinearities, however, are not likely to be important in nonprice data. 
estimates described above. Thus, to perform a parametric bootstrap, we draw $\varepsilon^{(\mathrm{i})} \sim \mathrm{N}\left(0, \mathrm{I}_{\mathrm{NT}}\right)$, we form

$$
\mathrm{Z}^{(\mathrm{i})}=\overline{\mathrm{z}}+\mathrm{P}^{*} \boldsymbol{\varepsilon}^{(\mathrm{i})} \sim \mathrm{N}\left(\overline{\mathrm{z}}, \Sigma^{*}\right),
$$

where $\bar{z}=1 \otimes \bar{y}$, and then we compute both the estimates $F^{*(i)}\left(\omega_{j}\right), j=1, \ldots, \frac{T}{2}-1, I=1$, ..., R and confidence intervals. Alternatively, to perform a nonparametric bootstrap, we note that $\varepsilon^{(\mathrm{i})}=\mathrm{P}^{-1}\left(\mathrm{z}-\mu_{\mathrm{z}}\right)$. In practice, we draw $\varepsilon^{(\mathrm{i})}$ with replacement from $\mathrm{P}^{*-1}(\mathrm{z}-\overline{\mathrm{z}})$, we form

$$
\mathrm{Z}^{(\mathrm{i})}=\overline{\mathrm{z}}+\mathrm{P}^{*} \boldsymbol{\varepsilon}^{(\mathrm{i})} \sim\left(\overline{\mathrm{z}}, \Sigma^{*}\right),
$$

from which we compute $\mathrm{F}^{*(\mathrm{i})}\left(\omega_{\mathrm{j}}\right), \mathrm{j}=1, \ldots, \frac{\mathrm{T}}{2}-1, \mathrm{I}=1, \ldots, \mathrm{R}$, and then we construct confidence intervals.

The simplicity of the Cholesky factor bootstrap is appealing. It is an intuitive and easily implemented generic method for generating samples of time series pseudo-data in which autocovariances match those of the actual data. Moreover, and perhaps surprisingly, the Cholesky factor bootstrap, which has a nonparametric flavor, and alternatives such as the VAR bootstrap (for example, in Canova, Finn, and Pagan, 1994), which has a parametric flavor, are in fact closely related. A modern and unifying view, currently the focus of intense research in mathematical statistics, is to interpret various time series bootstraps as sieves (Grenander, 1981) in which complexity increases with sample size at a suitable rate. ${ }^{5}$ The Cholesky factor bootstrap has a sieve interpretation. The sieve is a spectrum estimated by

\footnotetext{
${ }^{5}$ See, for example, Bickel and Bühlmann (1996) and Bühlmann (1997).
} 
smoothing a suitably increasing number of sample autocovariances as the sample size grows. The VAR bootstrap also has a sieve interpretation. The sieve is an estimated autoregression of suitably increasing length as the sample size grows. Thus, asymptotically in $\mathrm{T}$, both the Cholesky factor and VAR bootstraps can be effective algorithms for generating data with the same second-order properties as an observed sample path. Neither is in general superior to the other. The two are best viewed as complements. Both are the subject of ongoing research, as is the block bootstrap of Künsch (1989) and Liu and Singh (1992) as modified for spectra by Politis and Romano (1992), as well as the spectral bootstrap of Franke and Härdle (1992) and Berkowitz and Diebold (1997).

We hasten to add, however, that the literature on bootstrapping time series in generaland spectra in particular-is very young and very much unsettled. We still have a great deal to learn about the comparative properties of various bootstraps, both asymptotically and in finite samples, and the conditions required for various properties to obtain. Presently available results differ depending on the specific statistic being bootstrapped. Moreover, only scattered first- and occasionally second-order asymptotic results are available, and even less is known about actual finite-sample performance. With this in mind, we present both theoretical and Monte Carlo analyses of the performance of the Cholesky factor bootstrap in two appendixes to this paper, available at http://www.ssc.upenn.edu/ diebold/index.html. In Appendix 1, we establish first-order asymptotic validity, and in Appendix 2, we document good small-sample performance in a simple Monte Carlo experiment. 


\section{Constructing Confidence Tunnels}

For notational simplicity, we focus on confidence tunnels for univariate spectra. As will be clear, the extension to cross spectra is immediate. If interest centers on only one frequency, we simply use the bootstrap distribution at that frequency to construct the usual bootstrap confidence interval. That is, we find $\mathrm{q}_{\mathrm{T}}^{\mathrm{L}}, \mathrm{q}_{\mathrm{T}}^{\mathrm{U}}$ such that $\mathrm{P}\left(\mathrm{f}^{*(\cdot)}(\omega) \leq \mathrm{q}_{\mathrm{T}}^{\mathrm{U}}\right)=1-\frac{\alpha}{2}$

and $\mathrm{P}\left(\mathrm{f}^{*(.)}(\omega) \geq \mathrm{q}_{\mathrm{T}}^{\mathrm{L}}\right)=1-\frac{\alpha}{2}$, where $(1-\alpha)$ is the desired confidence level, $\mathrm{L}$ stands for lower, $\mathrm{U}$ stands for upper, the T subscript indicates that we tailor the band to the finite-sample size $\mathrm{T}$, and the (.) superscript indicates that we take the probability under the bootstrap distribution. The $(1-\alpha) \%$ two-sided confidence interval is $\left[\mathrm{q}_{\mathrm{T}}^{\mathrm{L}}, \mathrm{q}_{\mathrm{T}}^{\mathrm{U}}\right]$.

However, we may want to assess the sampling variability of the entire spectral density function over many frequencies (for example, business cycle frequencies or perhaps all frequencies) to learn about the broad agreement between model and data. One approach is to form the pointwise bootstrap confidence intervals described above and then to "connect the dots." But obviously, a set of $(1-\alpha) \%$ confidence intervals constructed for each of $n$ ordinates will not achieve $(1-\alpha) \%$ joint coverage probability. Rather, the actual confidence level will be closer to $(1-\alpha)^{\mathrm{n}} \%$, which holds exactly if the pointwise intervals are independent. A better approach is to use the Bonferroni method to approximate the desired coverage level by assigning $(1-\alpha / \mathrm{n}) \%$ coverage to each ordinate. ${ }^{6}$ The resulting confidence

${ }^{6}$ In the univariate case, typically $n=T / 2-1$. In the multivariate case, the question arises as to how wide to cast the net in forming confidence tunnels. We might view each element of the spectral density matrix in isolation, for example, in which case, each of the respective confidence tunnels would use $n=T / 2-1$. At the other extreme, we could use $\mathrm{n}=\mathrm{N}^{2}(\mathrm{~T} / 2-1)$, effectively forming a tunnel for the entire matrix. 
tunnel has coverage of at least $(1-\alpha) \%$ and therefore provides a conservative estimate of the tunnel.

A third approach to confidence tunnel construction is the supremum method of Woodroofe and van Ness (1967) and Swanepoel and van Wyk (1986), which uses an estimate of the (standardized) distribution of $\sup _{0<\omega_{j}<\pi}\left|f^{*}\left(\omega_{j}\right)-f\left(\omega_{j}\right)\right|, \omega_{j}=\frac{2 \pi j}{T}, j=1, \ldots, \frac{T}{2}-1$ to construct a confidence tunnel for the curve. Specifically,

(1) Calculate $\mathrm{f}^{*(\cdot)}\left(\omega_{\mathrm{j}}\right), \omega_{\mathrm{j}}=\frac{2 \pi \mathrm{j}}{\mathrm{T}}, \mathrm{j}=1, \ldots, \frac{\mathrm{T}}{2}-1$.

(2) Find c such that

$$
\mathrm{P}\left[\sup _{0<\omega_{\mathrm{j}}<\pi}\left(\frac{\left|\mathrm{f}^{*(.)}\left(\omega_{\mathrm{j}}\right)-\mathrm{f}^{*}\left(\omega_{\mathrm{j}}\right)\right|}{\sqrt{2 / \mathrm{T}} \mathrm{f}^{*}\left(\omega_{\mathrm{j}}\right)}\right) \leq \mathrm{c}\right]=1-\alpha
$$

where we evaluate the probability with respect to the bootstrap distribution.

(3) Construct the confidence tunnel, $\mathrm{f}^{*}\left(\omega_{\mathrm{j}}\right) \pm \mathrm{c} \sqrt{2 / \mathrm{T}} \mathrm{f}^{*}\left(\omega_{\mathrm{j}}\right)$,

$$
\omega_{\mathrm{j}}=\frac{2 \pi \mathrm{j}}{\mathrm{T}}, \mathrm{j}=1, \ldots, \frac{\mathrm{T}}{2}-1 .
$$

Unlike the Bonferroni tunnels, the supremum tunnels attain asymptotically correct coverage rates even with statistical dependence among ordinates. Little is known, however, about the comparative finite-sample performance of the Bonferroni and supremum tunnels, and the supremum tunnels may require very large samples for accurate coverage, as noted by Hannan (1970, p. 294). 


\section{Estimation: Maximizing Agreement Between Model and Data}

Now we consider estimation, together with the related issues of goodness-of-fit and hypothesis testing. To make the discussion as transparent as possible, we first discuss the univariate case, and then we proceed to the multivariate case.

$\underline{\text { Univariate }}$

Estimation requires a loss function, or goodness-of-fit measure, for assessing closeness between model and data. A strength of our approach is that many loss functions may be entertained. The particular loss function adopted should reflect the user's preferences, as argued by Pagan (1994). In most cases, it would seem that a function of the form

$$
\mathrm{C}_{\mathrm{gw}}(\theta)=\int_{0}^{\pi} \mathrm{g}\left(\mathrm{f}_{\mathrm{m}}(\omega ; \theta), \mathrm{f}^{*}(\omega)\right) \mathrm{w}(\omega) \mathrm{d} \omega
$$

would be adequate. The function $g$ measures the divergence between $f_{m}(\omega ; \theta)$ (the model spectrum) and $f^{*}(\omega)$ (an estimate of the data spectrum). Note that the model spectrum is analytically or numerically computable to any desired degree of accuracy and that the data spectrum, although unknown, is consistently estimable. We weight this divergence across frequencies by the function $\mathrm{w}(\omega)$. In practice, we replace the integral with a sum over frequencies $\omega_{j}=\frac{2 \pi j}{T}, j=1, \ldots, \frac{T}{2}-1$. Quadratic loss with uniform weighting over all frequencies, for example, corresponds to $g(a, b)=(a-b)^{2}$ and $w(\omega)=1$, yielding $\mathrm{C}_{\mathrm{gw}}(\theta)=\sum_{\mathrm{j}}\left(\mathrm{f}_{\mathrm{m}}\left(\omega_{\mathrm{j}} ; \theta\right)-\mathrm{f}^{*}\left(\omega_{\mathrm{j}}\right)\right)^{2}$.

The goodness-of-fit measure may readily be transformed into an estimation criterion by taking 
$\hat{\theta}_{\mathrm{gw}}=\underset{\theta}{\operatorname{argmin}} \mathrm{C}_{\mathrm{gw}}(\theta)$.

The Gaussian ML estimator is asymptotically of this form for a particular and potentially restrictive choice of $g, f^{*}$, and $w$. It is $\underset{\theta}{\operatorname{argmax}}\left(-\frac{1}{2} \sum_{\mathrm{j}} \ln \mathrm{f}_{\mathrm{m}}\left(\omega_{\mathrm{j}} ; \theta\right)-\frac{1}{2} \sum_{\mathrm{j}} \frac{\hat{\mathrm{f}}\left(\omega_{\mathrm{j}}\right)}{\mathrm{f}_{\mathrm{m}}\left(\omega_{\mathrm{j}} ; \theta\right)}\right)$. Note in particular that Gaussian ML involves minimization of spectral divergence with equal weighting across all frequencies.

To compute standard errors and interval estimates for parameters of interest and to test hypotheses about the elements of $\hat{\theta}_{\mathrm{gw}}$, we again use the Cholesky factor bootstrap. We proceed as follows:

(1) At bootstrap replication (i), draw a bootstrap sample of size T using the Cholesky factor algorithm.

(2) Numerically minimize $\mathrm{C}_{\mathrm{gw}}^{(\mathrm{i})}(\theta)$ to get $\hat{\theta}_{\mathrm{gw}}^{(\mathrm{i})}$.

(3) Repeat R times.

(4) Compute standard errors, form interval estimates, implement bias corrections, or test hypotheses using the distribution of $\hat{\theta}_{\mathrm{gw}}^{(\mathrm{i})}, \mathrm{i}=1, \ldots, \mathrm{R}$.

Note that implementation of the Cholesky factor bootstrap does not involve conditioning on the economic model; instead, the bootstrap samples are generated directly from the sample autocovariance matrix of the data. This is important in our environment, in which all models are best regarded as false.

In closing this section, let us elaborate on our allowance for differential weighting by frequency. There are at least two reasons for entertaining this possibility. One is that use of a 
loss function that weights differentially by frequency may be helpful in dealing with measurement error, which may not contaminate all frequencies equally. In such situations, it may be prudent to downweight those frequencies that are likely to be more contaminated by measurement error. The other reason is that use of a loss function that weights differentially by frequency may be important in misspecified models. For example, as discussed by Hansen and Heckman (1996), model misspecification may contaminate some frequencies more than others. Examples of this include potential contamination at seasonal frequencies, as in the work of Hansen and Sargent (1993) and Sims (1993). Watson (1993) also advocates the use of differential weighting in parameter estimation for the same reason, although he doesn't pursue the matter and notes that optimizing a loss function at particular frequencies corresponds to constructing an analog estimator along the lines of Manski (1988).

$\underline{\text { Multivariate }}$

The multivariate analog of our earlier loss function is

$$
\mathrm{C}_{\mathrm{GW}}(\theta)=\int_{0}^{\pi} \mathrm{G}\left(\mathrm{F}_{\mathrm{m}}(\omega ; \theta), \mathrm{F}^{*}(\omega)\right) \odot \mathrm{W}(\omega) \mathrm{d} \omega,
$$

where $\odot$ denotes component-by-component multiplication. The multivariate analog of our earlier univariate quadratic loss function, for example, is

$$
\begin{aligned}
& \mathrm{C}_{\mathrm{GW}}(\theta)=\sum_{\mathrm{j}} \operatorname{tr}\left(\mathrm{D}^{\prime}\left(\omega_{\mathrm{j}} ; \theta\right) \mathrm{D}\left(\omega_{\mathrm{j}} ; \theta\right)\right) \text {, where } \mathrm{D}\left(\omega_{\mathrm{j}} ; \theta\right)=\mathrm{F}_{\mathrm{m}}\left(\omega_{\mathrm{j}} ; \theta\right)-\mathrm{F}^{*}\left(\omega_{\mathrm{j}}\right), \omega_{\mathrm{j}}=\frac{2 \pi \mathrm{j}}{\mathrm{T}}, \\
& \mathrm{j}=1, \ldots, \frac{\mathrm{T}}{2}-1 .
\end{aligned}
$$

The estimation criterion function has the same form as in the univariate case,

$$
\hat{\theta}_{\mathrm{GW}}=\underset{\theta}{\operatorname{argmin}} \mathrm{C}_{\mathrm{GW}}(\theta),
$$


and the bootstrap approaches to computing standard errors, confidence intervals, and hypothesis testing parallel the univariate case precisely. Furthermore, as expected, the multivariate Gaussian ML estimator emerges as a special and potentially restrictive case. It is

$$
\underset{\theta}{\operatorname{argmax}}\left(-\frac{1}{2} \sum_{\mathrm{j}} \ln \left|\mathrm{F}_{\mathrm{m}}\left(\omega_{\mathrm{j}} ; \theta\right)\right|-\frac{1}{2} \operatorname{tr} \sum_{\mathrm{j}} \mathrm{F}_{\mathrm{m}}^{-1}\left(\omega_{\mathrm{j}} ; \theta\right) \hat{\mathrm{F}}\left(\omega_{\mathrm{j}}\right)\right) .
$$

It is worth emphasizing how all parts of the spectrum contribute to loss in the multivariate case. Consider, for example, a bivariate model (variables $\mathrm{x}$ and $\mathrm{y}$ ) under quadratic loss.

Then

$$
\mathrm{D}\left(\omega_{\mathrm{j}} ; \theta\right)=\left(\begin{array}{ll}
\mathrm{d}_{\mathrm{xx}}\left(\omega_{\mathrm{j}} ; \theta\right) & \mathrm{d}_{\mathrm{xy}}\left(\omega_{\mathrm{j}} ; \theta\right) \\
\mathrm{d}_{\mathrm{yx}}\left(\omega_{\mathrm{j}} ; \theta\right) & \mathrm{d}_{\mathrm{yy}}\left(\omega_{\mathrm{j}} ; \theta\right)
\end{array}\right),
$$

where

$$
\begin{gathered}
d_{x x}\left(\omega_{j} ; \theta\right)=f_{x x, m}\left(\omega_{j} ; \theta\right)-f_{x x}^{*}\left(\omega_{j}\right) \\
d_{y y}\left(\omega_{j} ; \theta\right)=f_{y y, m}\left(\omega_{j} ; \theta\right)-f_{y y}^{*}\left(\omega_{j}\right) \\
d_{x y}\left(\omega_{j} ; \theta\right)=f_{x y, m}\left(\omega_{j} ; \theta\right)-f_{x y}^{*}\left(\omega_{j}\right) \\
d_{y x}\left(\omega_{j} ; \theta\right)=f_{y x, m}\left(\omega_{j} ; \theta\right)-f_{y x}^{*}\left(\omega_{j}\right)=\overline{f_{x y, m}\left(\omega_{j} ; \theta\right)}-\overline{f_{x y}^{*}\left(\omega_{j}\right)}=\overline{d_{x y}}\left(\omega_{j} ; \theta\right) .
\end{gathered}
$$

Thus,

$$
\begin{aligned}
\operatorname{tr}\left(D^{\prime}\left(\omega_{j} ; \theta\right) D\left(\omega_{j} ; \theta\right)\right)= & {\left[d_{x x}^{2}\left(\omega_{j} ; \theta\right)+d_{x y}\left(\omega_{j} ; \theta\right) d_{y x}\left(\omega_{j} ; \theta\right)\right]+\left[d_{y y}^{2}\left(\omega_{j} ; \theta\right)+d_{x y}\left(\omega_{j} ; \theta\right) d_{y x}\left(\omega_{j} ; \theta\right)\right] } \\
& =d_{x x}^{2}\left(\omega_{j} ; \theta\right)+2\left|d_{x y}\left(\omega_{j} ; \theta\right)\right|^{2}+d_{y y}^{2}\left(\omega_{j} ; \theta\right)
\end{aligned}
$$




$$
\begin{aligned}
=\left[f_{x x, m}\left(\omega_{j} ; \theta\right)-f_{x x}^{*}\left(\omega_{j}\right)\right]^{2}+2\left[\operatorname{re}\left(f_{x y, m}\left(\omega_{j} ; \theta\right)\right)-\operatorname{re}\left(f_{x y}^{*}\left(\omega_{j}\right)\right)\right]^{2} \\
\quad+2\left[\operatorname{im}\left(f_{x y, m}\left(\omega_{j} ; \theta\right)\right)-\operatorname{im}\left(f_{x y}^{*}\left(\omega_{j}\right)\right)\right]^{2}+\left[f_{y y, m}\left(\omega_{j} ; \theta\right)-f_{y y}^{*}\left(\omega_{j}\right)\right]^{2} .
\end{aligned}
$$

This expression shows clearly how the goodness of fit of both univariate spectra, as well as both the real and imaginary parts of the cross spectrum, contribute to loss.

\section{Application: The U.S. Cattle Cycle}

In this section, we provide a detailed illustration of the use of our assessment and estimation techniques by applying them to an important model of the dynamics of beef cattle consumption and stock developed by Rosen, Murphy, and Scheinkman (RMS, 1994). The socalled cattle cycle documented, for example, in Mundlak and Huang (1996) is a well-known phenomenon in agricultural economics. This simple, yet rich, model allows us to illustrate clearly the application of all the tools in our framework. Moreover, our findings provide new insight into the RMS model and its agreement with the data.

$\underline{\text { The Data }}$

We use annual data on U.S. cattle consumption and stock for the years 1900-1989, kindly supplied by Sherwin Rosen and originally obtained from Historical Statistics: Colonial Times to 1970 and Agricultural Statistics, published by the U.S. Department of Agriculture. We plot the series in Figures 1 and 2. The cycle is visually apparent. Moreover, the series are clearly trending. Following RMS, we remove a linear trend from each series prior to 
additional analysis, allowing for a break in the slope of the trend in 1930 . We also show the fitted trends in Figures 1 and 2.

We obtain the estimated data spectrum, presented in Figure 3, by smoothing the sample autocovariance function using a Bartlett window with truncation lag 24. We make use here and throughout of a matrix graphic with univariate spectra plotted on the main diagonal, coherence in the upper right corner, and phase in the lower left corner. In addition, following standard practice, we graph log spectra rather than spectra throughout and refer to the log spectra simply as spectra. Not all frequencies are of equal interest, however. The frequencies most relevant to an investigation of the cattle cycle, typically thought to have a period of roughly ten years, are not those in the entire $[0, \pi]$ range, but rather those in a subset that excludes very low and very high frequencies. This presents no problem for our procedures and in fact provides a good opportunity to illustrate the ease with which our procedures can be tailored to study specific applications. Thus, for much of our analysis, we concentrate on the frequency band corresponding to periods of 30 years to 4 years, indicated by the shaded region in Figure 3 and subsequent figures.

Four features of the point estimates of the data spectrum stand out. First, the consumption spectrum (and to a lesser extent, the stock spectrum) displays a power concentration at roughly a ten-year cycle. Second, both the consumption and stock spectra otherwise have Granger's (1966) typical spectral shape, with high power at low frequencies and declining power throughout the frequency range. Third, the coherence between consumption and stock is generally high and varies across frequencies, with a maximum (about .85) at roughly a ten-year cycle. Finally, the phase shift, measured in years by which 
consumption leads stock, varies with frequency. Within the band of interest, the maximum phase shift of roughly one year again occurs at a ten-year cycle.

In Figure 4, we present the data spectrum along with $90 \%$ confidence tunnels computed by using the conservative Bonferroni technique in conjunction with the Cholesky factor bootstrap. All of the point estimates display substantial uncertainty, as manifest in the wide $90 \%$ confidence tunnels. Such uncertainty associated with estimated spectra is typical of economic time series, although such uncertainty often goes unacknowledged.

The Model

We begin with some accounting identities. The head count of all animals $\left(y_{t}\right)$ is the sum of the adult breeding stock $\left(\mathrm{x}_{\mathrm{t}}\right)$, the stock of calves (assumed equal to $\mathrm{gx}_{\mathrm{t}-1}$ ), and the stock of yearlings (assumed equal to $\mathrm{gx}_{\mathrm{t}-2}$ ), where $\mathrm{g}$ is a fertility parameter. That is,

$$
y_{t}=x_{t}+g x_{t-1}+g x_{t-2}
$$

The adult breeding stock consists of surviving stock from the previous period (assumed equal to $\left.(1-\delta) \mathrm{x}_{\mathrm{t}-1}\right)$ and the yearlings from $\mathrm{t}-1$ entering the adult herd $\left(\mathrm{gx}_{\mathrm{t}-3}\right)$ less the number that are marketed $\left(\mathrm{c}_{\mathrm{t}}\right)$,

$$
x_{t}=(1-\delta) x_{t-1}+g x_{t-3}-c_{t}
$$

We are concerned with the equilibrium determination of $c_{t}$ and $y_{t}$. The risk-neutral rancher maximizes the present discounted value of expected profits, which involves equating the expected marginal benefit of marketing an animal for consumption to the expected marginal benefit of holding the animal for breeding. First, suppose that the rancher markets 
the animal for consumption. The rancher receives net revenue $q_{t}=p_{t}-m_{t}$, where $p_{t}$ is price and $\mathrm{m}_{\mathrm{t}}$ is finishing cost. Alternatively, suppose the rancher holds an animal for breeding. Expected discounted net revenue is the sum of expected discounted revenue from selling tomorrow plus expected discounted revenue from marketing the animal's offspring less expected total holding costs $\left(\mathrm{z}_{\mathrm{t}}\right), \mathrm{E}_{\mathrm{t}}\left[\beta(1-\delta) \mathrm{q}_{\mathrm{t}+1}+\beta^{3} \mathrm{gq}_{\mathrm{t}+3}-\mathrm{z}_{\mathrm{t}}\right]$. Total holding cost equals the sum of time $t$ holding costs $\left(h_{t}\right)$, discounted holding costs of the resultant time $t+1$ calves, and discounted holding costs of the resultant time $t+2$ yearlings. That is, $\mathrm{z}_{\mathrm{t}}=\mathrm{h}_{\mathrm{t}}+\beta \mathrm{g} \gamma_{0} \mathrm{~h}_{\mathrm{t}+1}+\beta^{2} \mathrm{~g} \gamma_{1} \mathrm{~h}_{\mathrm{t}+2}$ (when we assume proportional costs for calves and yearlings, $\gamma_{0}$ and $\left.\gamma_{1}\right)$.

In equilibrium, the expected marginal net revenue from marketing for consumption equals the expected marginal discounted net revenue from holding for breeding; that is,

$$
\mathrm{E}_{\mathrm{t}}\left[\mathrm{q}_{\mathrm{t}}\right]=\mathrm{E}_{\mathrm{t}}\left[\beta(1-\delta) \mathrm{q}_{\mathrm{t}+1}+\beta^{3} \mathrm{gq}_{\mathrm{t}+3}-\mathrm{z}_{\mathrm{t}}\right]
$$

We close the model by specifying the exogenous processes $\left\{\mathrm{m}_{\mathrm{t}}, \mathrm{h}_{\mathrm{t}}, \mathrm{d}_{\mathrm{t}}\right\}$ as first-order autoregressions. ${ }^{7}$ Following RMS, we assume that each of the three shocks has common serial-correlation parameter $\rho$.

The model structure implies that the reduced-form equations for $\mathrm{c}_{\mathrm{t}}$ and $\mathrm{y}_{\mathrm{t}}$ can be expressed in terms of a single disturbance, $\omega_{\mathrm{t}}$, which is a linear combination of the independent innovations from the three $\mathrm{AR}(1)$ driving processes. In particular, $\mathrm{c}_{\mathrm{t}} \sim \operatorname{ARMA}(2,1)$ and $\mathrm{y}_{\mathrm{t}} \sim \operatorname{ARMA}(4,2):$

${ }^{7}$ We denote the preference shock by $d_{t}$. We have not discussed the demand side of the model, because we do not use it in estimation. 


$$
\begin{gathered}
\left(1-\lambda_{1} \mathrm{~L}\right)(1-\rho \mathrm{L}) \mathrm{c}_{\mathrm{t}}=-\left(1-\phi_{1} \mathrm{~L}\right) \omega_{\mathrm{t}} \\
\left(1-\lambda_{1} \mathrm{~L}\right)\left(1-\phi_{2} \mathrm{~L}\right)\left(1-\phi_{3} \mathrm{~L}\right)(1-\rho \mathrm{L}) \mathrm{y}_{\mathrm{t}}=\left(1+\mathrm{gL}+\mathrm{gL}^{2}\right) \omega_{\mathrm{t}},
\end{gathered}
$$

where $\phi_{1}$ is the one unstable root and $\left\{\phi_{2}, \phi_{3}\right\}$ are the two stable roots of

$$
\phi^{3}-(1-\delta) \phi^{2}-\mathrm{g}=0
$$

and $\lambda_{1}$ is the one stable root of

$$
\mathrm{g} \beta^{3} \lambda^{3}+(1-\delta) \beta \lambda-1=0
$$

The associated univariate spectra are

$$
\begin{gathered}
\mathrm{f}_{\mathrm{c}}(\omega)=\sigma_{\omega}^{2} \frac{\left|\left(1-\phi_{1} \mathrm{e}^{\mathrm{i} \omega}\right)\right|^{2}}{\left|\left(1-\lambda_{1} \mathrm{e}^{\mathrm{i} \omega}\right)\left(1-\rho \mathrm{e}^{\mathrm{i} \omega}\right)\right|^{2}} \\
\mathrm{f}_{\mathrm{y}}(\omega)=\sigma_{\omega}^{2} \frac{\left|\left(1+g \mathrm{e}^{\mathrm{i} \omega}+\mathrm{ge}^{2 \mathrm{i} \omega}\right)\right|^{2}}{\left|\left(1-\lambda_{1} \mathrm{e}^{\mathrm{i} \omega}\right)\left(1-\phi_{2} \mathrm{e}^{\mathrm{i} \omega}\right)\left(1-\phi_{3} \mathrm{e}^{\mathrm{i} \omega}\right)\left(1-\rho \mathrm{e}^{\mathrm{i} \omega}\right)\right|^{2}},
\end{gathered}
$$

and the cross spectrum is

$$
\mathrm{f}_{c y}(\omega)=\frac{-\left(1-\phi_{1} \mathrm{e}^{\mathrm{i} \omega}\right)\left(1-\phi_{2} \mathrm{e}^{\mathrm{i} \omega}\right)\left(1-\phi_{3} \mathrm{e}^{\mathrm{i} \omega}\right)}{\left(1+\mathrm{ge}^{\mathrm{i} \omega}+\mathrm{ge}^{2 \mathrm{i} \omega}\right)} \mathrm{f}_{\mathrm{y}}(\omega) .
$$

These equations provide a full description of the model in the frequency domain. The variance $\sigma_{\omega}^{2}$ is a complicated function of the structural parameters, including some from the demand side of the model. All of the parameters of present interest, however, may be 
identified from the other reduced-form parameters, with the exception of $\gamma_{0}$ and $\gamma_{1}$. We therefore treat $\sigma_{\omega}^{2}$ as a free parameter and estimate it subject to no restrictions.

RMS do not estimate the cattle cycle model. Rather, they choose values for the behavioral parameters and report that the calibrated model fits the data well. In the following sections, we explicitly estimate the model and assess its performance.

Estimating the Model

Estimation requires specification of an explicit loss function. We use a loss function that incorporates the focus in the cattle cycle literature on cycles of roughly 10 years. We exclude frequencies corresponding to periods of more than 30 years or less than 4 years, which from the standpoint of our earlier discussion of frequency downweighting corresponds to giving equal weight to frequencies in the band of interest and giving zero weight to frequencies outside the band. Minimization of such a loss function, which measures divergence between model and data spectra only within a particular frequency band, leads us to an estimator that we call band-restricted maximum likelihood (band-ML). ${ }^{8}$

We perform the minimization using the simplex algorithm, which is a derivative-free method, as implemented in the Matlab fmins.m procedure. Using penalty functions, we constrain the discount factor to be between 0.65 and 1.00 , the fertility rate to be between 0.00 and 1.00 , the death rate to be between 0.00 and 1.00 , the persistence parameter to be between 0.00 and 1.00 , and the scale parameter to be between 0.10 and 7.00 . We start the iterations with the RMS parameter values for the discount rate, fertility rate, death rate, and persistence

${ }^{8}$ Gaussian band-ML is the maximum-likelihood analog of Engle's (1974) band-spectral linear regression. Band-ML may, of course, be undertaken for models more complicated than simple linear regression, such as the present one. 
parameter. RMS do not report a value for the scale parameter, which we start at 1.7. In our experience, estimation is numerically straightforward and stable. The estimated parameter vector is always in the interior of the constraint set, convergence is fast, and alternative starting values produce virtually identical estimates. ${ }^{9}$

We display the band-ML estimates in Table 1. For comparison, we also display the RMS parameter values. Several of the band-ML parameter estimates are similar to those chosen by RMS. In particular, the estimate of the death rate parameter (0.08) is nearly identical to the RMS value (0.10), the estimate of the producer's discount factor $(0.86)$ is close to the RMS value (0.91), and the estimated fertility parameter $(0.67)$ is lower than, but nevertheless close to, the RMS value (0.85). As stressed in Diebold (1998), however, even small differences in parameters can make important differences for activities such as forecasting.

The band-ML estimate of the persistence parameter, however, differs substantially from the RMS value. RMS choose a fairly persistent value of 0.6 . In contrast, we find that optimizing the band-ML loss function requires very little persistence in the driving process (0.2). This implies that the RMS model has a strong internal propagation mechanism: the model takes shocks with relatively little serial correlation and transforms them into series that display substantial persistence in equilibrium. This dimension of the RMS model differs

${ }^{9}$ One wrinkle does arise: simple economic models such as this one, in which one shock drives the evolution of a higher-dimensional system, have a singular spectral density $\mathrm{F}_{\mathrm{m}}$. This presents a problem for implementation of the Gaussian ML and band-ML estimators, which involve $\mathrm{F}_{\mathrm{m}}^{-1}$. We have found that the problem can be satisfactorily skirted in practice, because the model spectrum is typically obtained by simulating a long realization from the model, in which the spectrum is then consistently estimated. This has the effect of introducing just enough measurement error to avoid a singular spectrum. 
fundamentally from standard dynamic equilibrium models used in macroeconomics, international economics, and public finance. As Watson (1993) and others have noted, models used in those fields typically have weak internal propagation mechanisms-they require highly persistent underlying shocks to generate a realistic amount of serial correlation in the variables determined in equilibrium.

In addition to finding the parameter estimates that maximize agreement between model and data, we can assess the sampling uncertainty of those estimates within our framework. Standard errors are of some use in that regard, in spite of the fact that the sampling distributions need not be Gaussian. We compute standard errors using 200 replications of the Cholesky factor bootstrap procedure, and we report them in parentheses below the estimated parameters in Table 1. More generally, our bootstrap procedures allow us to estimate the entire sampling distributions of the estimated parameters. We report in them in Figure 5. The estimated sampling distributions of the discount factor, the depreciation rate, and the persistence parameter are fairly concentrated, while the estimated sampling distribution of the fertility rate is more dispersed.

Our framework also enables us to examine the joint distribution of the estimated parameters. In Table 2, we present bootstrap estimates of the correlations between the estimated parameters. Perhaps the most interesting relationship is the strong negative correlation between the discount factor and the fertility rate, which occurs because the discount factor and the fertility rate enter multiplicatively in one of the cubic equations that define the ARMA polynomials. This implies that the loss function trades off high fertility 
rates for low discount factors and suggests that fixing either one of the parameters at the higher RMS value would tend to result in an even lower estimate for the other.

\section{Assessing the Model}

To assess divergence between model and data, we plot the model spectrum evaluated at the band-ML parameter estimates in Figure 6, together with the earlier-discussed point estimates and $90 \%$ confidence tunnels for the data spectrum, produced with 200 replications of the nonparametric Cholesky factor bootstrap. The diagonal elements of the figure provide comparative assessments of model and data univariate dynamics, and the off-diagonal elements provide comparative assessments of cross-variable dynamics.

Given the objective of constructing a simple model that is consistent with periodic behavior in consumption and stock, a surprising finding is that neither the consumption nor the stock model spectrum has a peak corresponding to a ten-year cycle, which suggests that the model does not easily produce cyclical behavior at the band-ML parameter values. Moreover, the rate of decay of the model consumption spectrum appears to be slower than that of the data spectrum. Thus, although the model and data consumption spectra are insignificantly different over most of the relevant frequency range, they begin to deviate significantly for cycles with periods of four years or less. Conversely, the rate of decay of the model stock spectrum appears to be significantly faster than that of the data spectrum. The two diverge not only at high frequencies, but also over much of the relevant frequency range. In particular, the model stock spectrum lies slightly outside the lower region of the $90 \%$ confidence tunnel for cycles of about 20 years and less. 
Now let us examine phase and coherence. As with the model spectra, the model phase shift declines monotonically, which contrasts somewhat with the point estimate of the data phase shift, which has a local peak at roughly the ten-year cycle. In addition, the model generates significantly more phase shift than we see in the data, particularly at the low and middle frequencies of interest. Finally, the model coherence reminds us of an inherent limitation of the model: because it is driven by a single shock, the model is singular, which forces unit coherence at all frequencies. Thus, the model and data coherence diverge sharply. In spite of the fact that the confidence tunnel is very wide, the unit model coherence is always outside the data coherence confidence tunnel.

We also show in Figure 6 the model spectrum evaluated at the RMS parameter values. The major difference between the two parameterizations of the model, within the frequency band of interest, is revealed in the spectral density of consumption. The RMS parameters generate substantially more variation at low and middle frequencies than do the band-ML parameters. The differences in the phase shift and the spectral density of stock across the two parameterizations of the model are modest.

All told, comparison of the band-ML and RMS parameters, and model spectra evaluated at those parameters, reveals some similarities and some differences, with the similarities outnumbering the differences. The real insight afforded by our analysis is that the similar model behavior across different parameterizations is due to several model deficiencies and that the deficiencies can't be remedied simply by exploring different estimators. Rather, they need to be remedied at the source by improving the model. We hope that our results stimulate such work. An appropriately improved model might include richer dynamics for 
driving processes, incorporation of a demand side and prices, and explicit determination of trend.

\section{Conclusions and Directions for Future Research}

We have described a framework for evaluating dynamic economic models that should be useful to applied economists in many fields. The framework is flexible-it can be used by researchers to evaluate purely calibrated models, and it can also be used by researchers interested in estimating parameters and conducting inference. The framework's frequencydomain foundations provide useful diagnostics that nicely complement alternative time-domain approaches. In particular, the framework is graphical and constructive, and it takes seriously several important issues in the quantitative analysis of simple dynamic equilibrium models: model misspecification, the user's objectives, and small sample sizes.

Our analysis of the RMS model of cattle cycles illustrates the use of our tools for formally estimating models and performing statistical inference as well as for assessing agreement between models and data at various parameter values, whether preset or estimated. In addition, our analysis sheds light on the characteristics of the RMS model and, in particular, its strong internal propagation mechanism. Our analysis also reveals that the model is deficient in several key respects, not the least of which is its inability to generate internal spectral peaks.

The ultimate goal of the research program of which this paper is a part is to facilitate communication between researchers with potentially very different research objectives and strategies, thereby bringing modern dynamic economic theory into closer and more frequent contact with dynamic economic data. As economists use richer and more complicated models 
to understand a wider variety of data, we hope that our framework will find use in discerning the dimensions along which models are consistent—and inconsistent—with data. That information can in turn be used to help construct new and improved models. 


\section{References}

Auerbach, A.J. and Kotlikoff, L.J. (1987), Dynamic Fiscal Policy. Cambridge: Cambridge University Press.

Backus, D.K., Kehoe, P.J. and Kydland, F.E. (1994), "Dynamics of the Trade Balance and the Terms of Trade: The J-Curve?" American Economic Review, 84, 84-103.

Berkowitz, J. and Diebold, F.X. (1997), "Bootstrapping Multivariate Spectra,” Manuscript, Department of Economics, University of Pennsylvania, http://www.ssc.upenn.edu/ diebold/index.html.

Bickel, P. and Bühlmann, P. (1996), "Mixing Property and Functional Central Limit Theorems for a Sieve Bootstrap in Time Series," Manuscript, Department of Statistics, University of California, Berkeley.

Bühlmann, P. (1997), “Sieve Bootstraps for Time Series,” Bernoulli, in press.

Canova, F., Finn, M., and Pagan, A.R. (1994), "Evaluating a Real Business Cycle Model," in C.P. Hargreaves (ed.), Nonstationary Time Series and Cointegration. Oxford: Oxford University Press, p. 225-255.

Christiano, L.J. and Eichenbaum, M. (1995), "Liquidity Effects, Monetary Policy and the Business Cycle," Journal of Money, Credit and Banking, 27, 1113-1136.

Cogley, T. and Nason, J.M. (1995), "Output Dynamics in Real Business Cycle Models," American Economic Review, 85, 492-511.

Cooley, T.F. and Hansen, G.D. (1992), "Tax Distortions in a Neoclassical Monetary Economy," Journal of Economic Theory, 58, 290-316.

Deaton, A. and Laroque, G. (1995), "Estimating a Nonlinear Rational Expectations Commodity Price Model with Unobservable State Variables," Journal of Applied Econometrics, 10, 9-40.

Diebold, F.X. (1998), "The Past, Present and Future of Macroeconomic Forecasting," Journal of Economic Perspectives, 12, in press.

Engle, R.F. (1974), "Band Spectrum Regression," International Economic Review, 15, 1-11.

Ericson, R. and Pakes, A. (1995), "Markov-Perfect Industry Dynamics: A Framework for Empirical Work," Review of Economic Studies, 62, 53-82. 
Franke, J. and Härdle, W. (1992), “On Bootstrapping Kernel Spectral Estimates,” Annals of Statistics, 20, 121-145.

Granger, C.W.J. (1966), “The Typical Spectral Shape of an Economic Variable," Econometrica, 34, 150-161.

Greenwood, J., Hercowitz, Z. and Krusell, P. (1997), “Long Run Implications of Equipment-Specific Technological Change," American Economic Review, in press.

Gregory, A.W. and Smith, G.W. (1991), "Calibration as Testing: Inference in Simulated Macroeconomic Models," Journal of Business and Economic Statistics, 9, 297-303.

Grenander, U. (1981), Abstract Inference. New York: John Wiley.

Hannan, E.J. (1970), Multiple Time Series. New York: John Wiley.

Hansen, G.D. (1985), "Indivisible Labor and the Business Cycle," Journal of Monetary Economics, 16, 309-327.

Hansen, L.P and Heckman, J.J. (1996), “The Empirical Foundations of Calibration,” Journal of Economic Perspectives, 10, 87-104.

Hansen, L.P., McGrattan, E. and Sargent, T.J. (1997), "Mechanics of Forming and Estimating Dynamic Linear Economies," in J. Rust, D. Kendrick, and H. Amman (eds.), Handbook of Computational Economics, in press.

Hansen, L.P. and Sargent, T.J. (1993), "Seasonality and Approximation Errors in Rational Expectations Models," Journal of Econometrics, 55, 21-55.

Jones, L.E. and Manuelli, R.E. (1990), “A Convex Model of Equilibrium Growth: Theory and Policy Implications," Journal of Political Economy, 98, 1008-1038.

Kim, K. and Pagan, A.R. (1994), “The Econometric Analysis of Calibrated Macroeconomic Models," in H. Pesaran and M. Wickens (eds.), Handbook of Applied Econometrics. Oxford: Blackwell.

King, R.G. and Watson, M.W. (1992), "On the Econometrics of Comparative Dynamics," Manuscript, Departments of Economics, University of Rochester and Northwestern University.

King, R.G. and Watson, M.W. (1996), "Money, Prices, Interest Rates and the Business Cycle," Review of Economics and Statistics, 78, 35-53. 
Künsch, H.R. (1989), “The Jackknife and the Bootstrap for General Stationary Observations," Annals of Statistics, 17, 1217-1241.

Kydland, F.E. and Prescott, E.C. (1982), "Time to Build and Aggregate Fluctuations," Econometrica, 50, 1345-1370.

Kydland, F.E. and Prescott, E.C. (1996), "The Computational Experiment: An Econometric Tool," Journal of Economic Perspectives, 10, 69-86.

Leeper, E.M. and Sims, C.A. (1994), "Toward a Modern Macroeconomic Model Usable for Policy Analysis," in S. Fischer and J.J. Rotemberg (eds.), NBER Macroeconomics Annual. Cambridge, Mass.: MIT Press, pp. 81-118.

Liu, R.Y. and Singh, K. (1992), "Moving Blocks Jacknife and Bootstrap Capture Weak Dependence," in R. LePage and L. Billard (eds.), Exploring the Limits of the Bootstrap. New York: John Wiley.

Lucas, R.E., Jr. (1977), “Understanding Business Cycles,” Journal of Monetary Economics, $5,7-29$.

Lucas, R.E., Jr. (1988), “On The Mechanics of Economic Development,” Journal of Monetary Economics, 22, 3-42.

Lucas, R.E., Jr. (1990), "Supply Side Economics: An Analytical Review,” Manuscript, Department of Economics, University of Chicago.

Manski, C.F. (1988), Analog Estimation Methods in Econometrics. New York: Chapman and Hall.

Mundlak, Y. and Huang, H. (1996), "International Comparisons of Cattle Cycles," American Journal of Agricultural Economics, 78, 855-868.

Ohanian, L.E. (1997), "The Macroeconomic Effects of War Finance in the United States: World War II and the Korean War," American Economic Review, 87, 23-40.

Pagan, A. (1994), "Calibration and Econometric Research: An Overview: Introduction," in A. Pagan (ed.), Calibration Techniques and Econometrics, special issue of Journal of Applied Econometrics, 9, S1-S10.

Politis D.N. and Romano J.P. (1992), “A General Resampling Scheme for Triangular Arrays of $\alpha$-Mixing Random Variables with an Application to the Problem of Spectral Density Estimation," Annals of Statistics, 20, 1985-2007. 
Ramos, E. (1988), "Resampling Methods for Time Series," Technical Report ONR-C-2, Department of Statistics, Harvard University.

Rebelo, S. (1991), "Long-Run Policy Analysis and Long-Run Growth," Journal of Political Economy, 99, 500-521.

Rosen, S., Murphy, K.M. and Scheinkman, J.A. (1994), "Cattle Cycles," Journal of Political Economy, 102, 468-492.

Rotemberg, J.J. and Woodford, M. (1996), "Real-Business-Cycle Models and the Forecastable Movements in Output, Hours, and Consumption," American Economic Review, 86, 71-89.

Rotemberg, J.J. and Woodford, M. (1997), “An Optimization-Based Econometric Framework for the Evaluation of Monetary Policy," in O. Blanchard and S. Fischer (eds.), NBER Macroeconomics Annual. Cambridge, Mass.: MIT Press.

Rust, J. (1989), “A Dynamic Programming Model of Retirement Behavior,” in D.A. Wise (ed.), The Economics of Aging. Chicago: University of Chicago Press, pp. 359-398.

Sims, C.A. (1993), "Rational Expectations Modeling with Seasonally Adjusted Data," Journal of Econometrics, 55, 9-19.

Sims, C.A. (1996), "Macroeconomics and Methodology," Journal of Economic Perspectives, $10,105-120$.

Swanepoel, J.W.H. and van Wyk, J.W.J. (1986), "The Bootstrap Applied to Power Spectral Density Function Estimation," Biometrika, 73, 135-141.

Watson, M.W. (1993), "Measures of Fit for Calibrated Models," Journal of Political Economy, 101, 1011-1041.

Woodroofe, M.B. and van Ness, J.W. (1967), "The Maximum Deviation of Sample Spectral Densities," Annals of Mathematical Statistics, 38, 1559-1569. 
Table 1

Parameter Estimates

Band-Restricted Maximum Likelihood Estimation

\begin{tabular}{cccccc}
\hline \hline Parameter: & $\beta$ & $\mathrm{g}$ & $\delta$ & $\rho$ & $\sigma_{\omega}^{2}$ \\
\hline \hline
\end{tabular}

Estimation or

Calibration Method

$\begin{array}{rlllll}\text { Band-ML } & .86 & .67 & .08 & .21 & 2.10 \\ & (.03) & (.09) & (.03) & (.10) & (.37) \\ \text { RMS } & & & & & \\ & .909 & .85 & .10 & .60 & \text { NA } \\ & \text { (NA) } & \text { (NA) } & \text { (NA) } & \text { (NA) } & \text { (NA) }\end{array}$

Notes to Table: $\beta$ is the discount factor, $g$ is the fertility rate, $\delta$ is the death rate, and $\rho$ is the persistence parameter. Band-ML denotes the band-restricted maximum likelihood estimation, with the frequency band used for estimation corresponding to periods of 30 to 4 years. Standard errors, based on 200 bootstrap replications, appear in parentheses. RMS denotes the RosenMurphy-Scheinkman calibrated parameters. (They have no standard errors, because they were not estimated.)

Table 2

Estimated Parameter Correlations

Band-Restricted Maximum Likelihood Estimation

\begin{tabular}{lllll}
\hline \hline & $\beta$ & $\mathrm{g}$ & $\delta$ & $\rho$ \\
\hline \hline$\beta$ & 1.00 & & & \\
$\mathrm{~g}$ & -.73 & 1.00 & & \\
$\delta$ & .49 & -.37 & 1.00 & \\
$\rho$ & -.19 & .10 & .06 & 1.00 \\
\hline \hline
\end{tabular}

Notes to Table: $\beta$ is the discount factor, $g$ is the fertility rate, $\delta$ is the death rate, and $\rho$ is the persistence parameter. Estimated parameter correlations are based on 200 bootstrap replications. The frequency band used for estimation corresponds to periods of 30 to 4 years. 
Figure 1

U.S. Cattle Consumption, 1900-1990

Actual and Estimated Trend

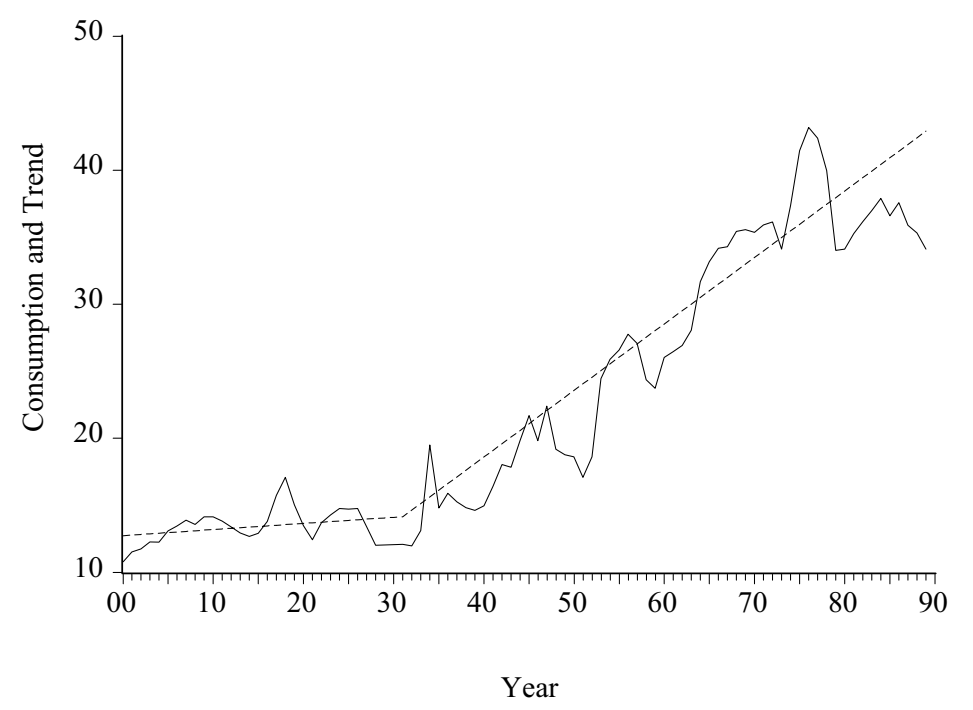

Notes to Figure: We show cattle consumption (solid line) and the estimated kinked-linear trend (dashed line).

Figure 2

U.S. Cattle Stock, 1900-1990

Actual and Estimated Trend

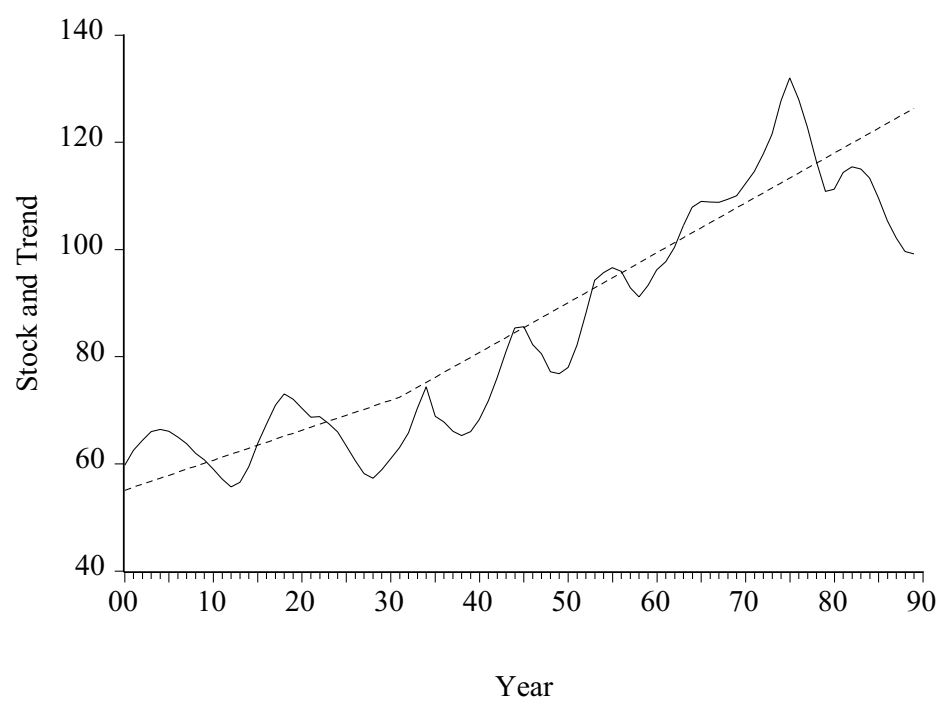

Notes to Figure: We show cattle stock (solid line) and the estimated kinked-linear trend (dashed line). 


\section{Figure 3}

Estimated Spectral Density Matrix

U.S. Cattle Consumption and Stock

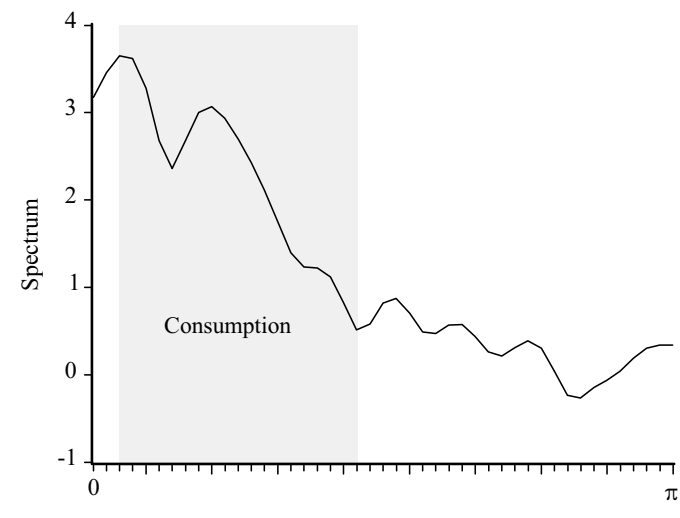

Frequency

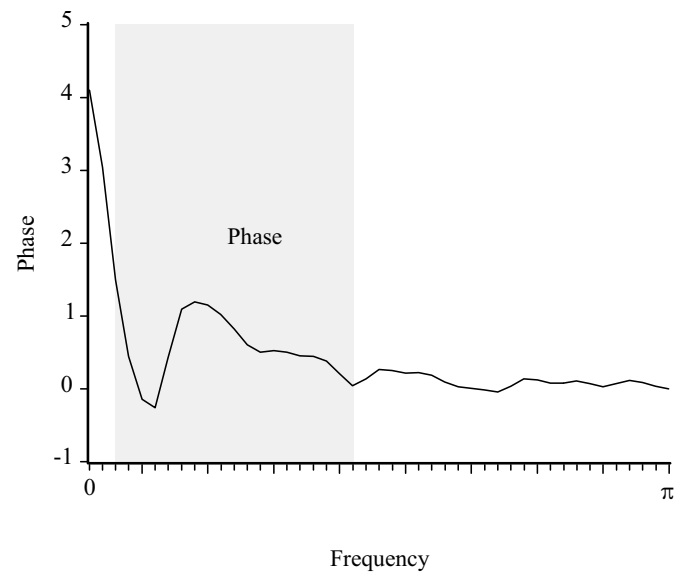

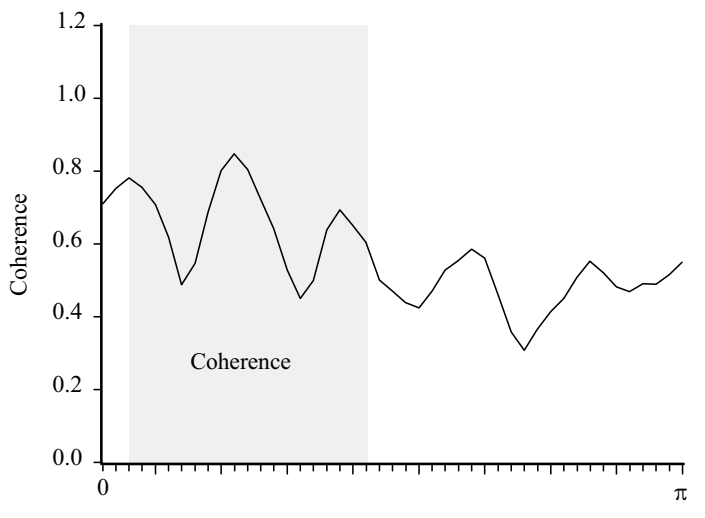

Frequency

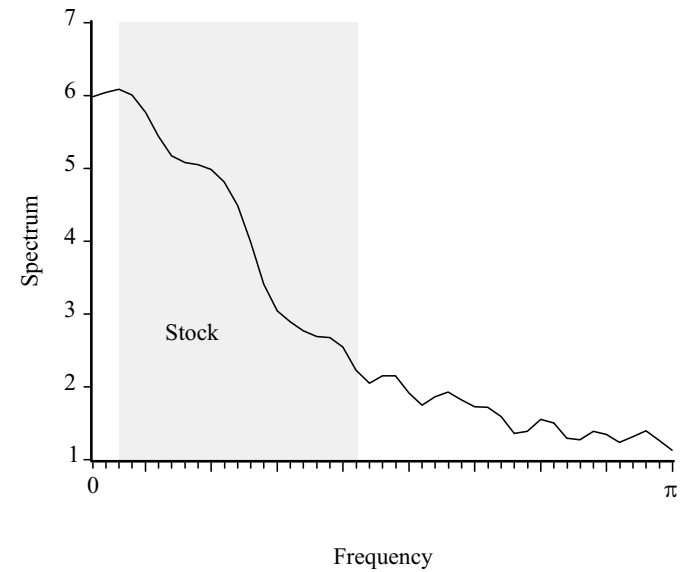

Notes to Figure: We show the point estimate of each element of the spectral density matrix. The shaded frequency band corresponds to cycles with periods of 30 to 4 years. 
Figure 4

Estimated Spectral Density Matrix and Confidence Tunnels

U.S. Cattle Consumption and Stock
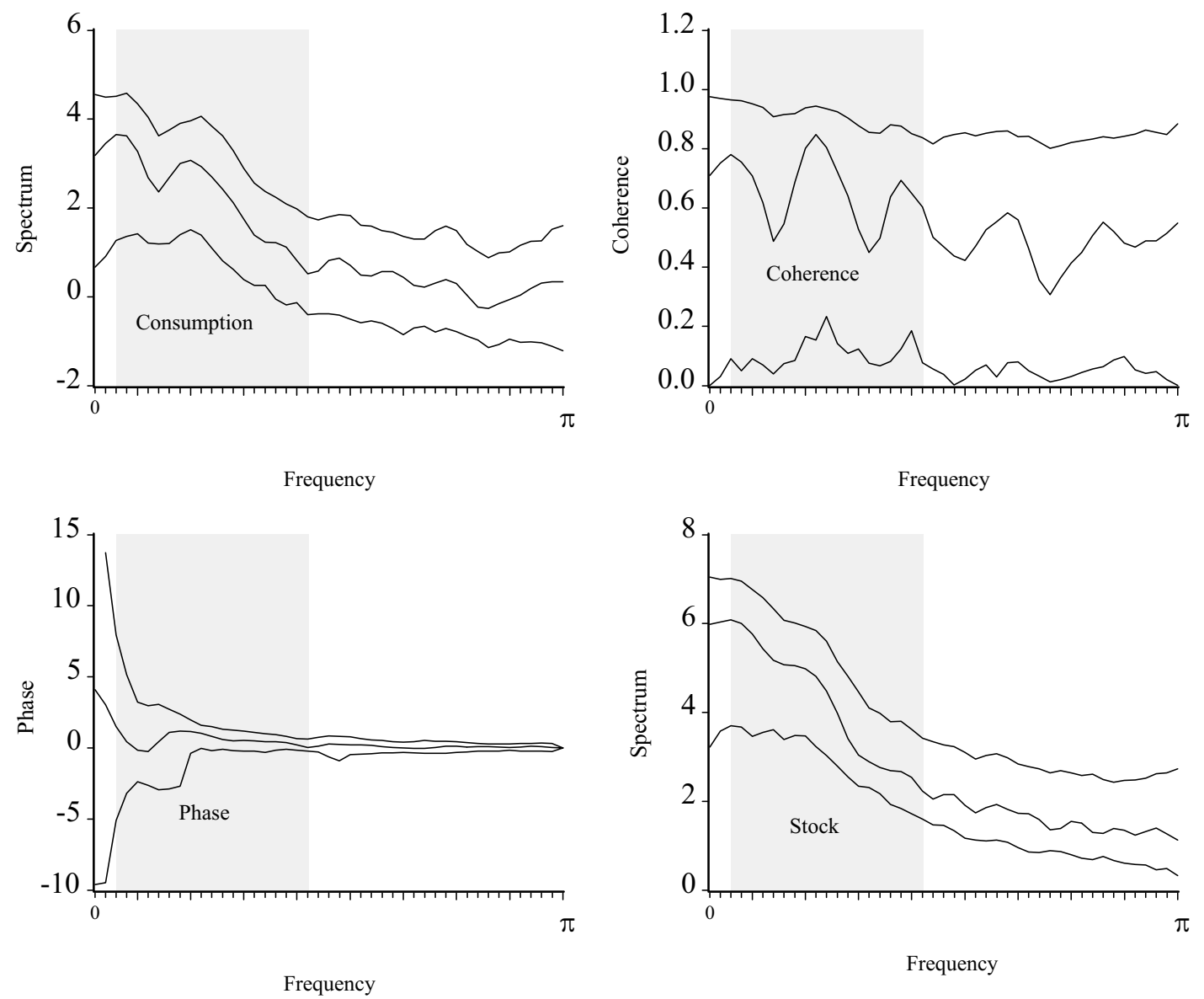

Notes to Figure: We show the point estimate together with a $90 \%$ confidence tunnel for each element of the spectral density matrix. The shaded frequency band corresponds to cycles with periods of 30 to 4 years. 
Figure 5

Bootstrap Estimates of Sampling Distributions
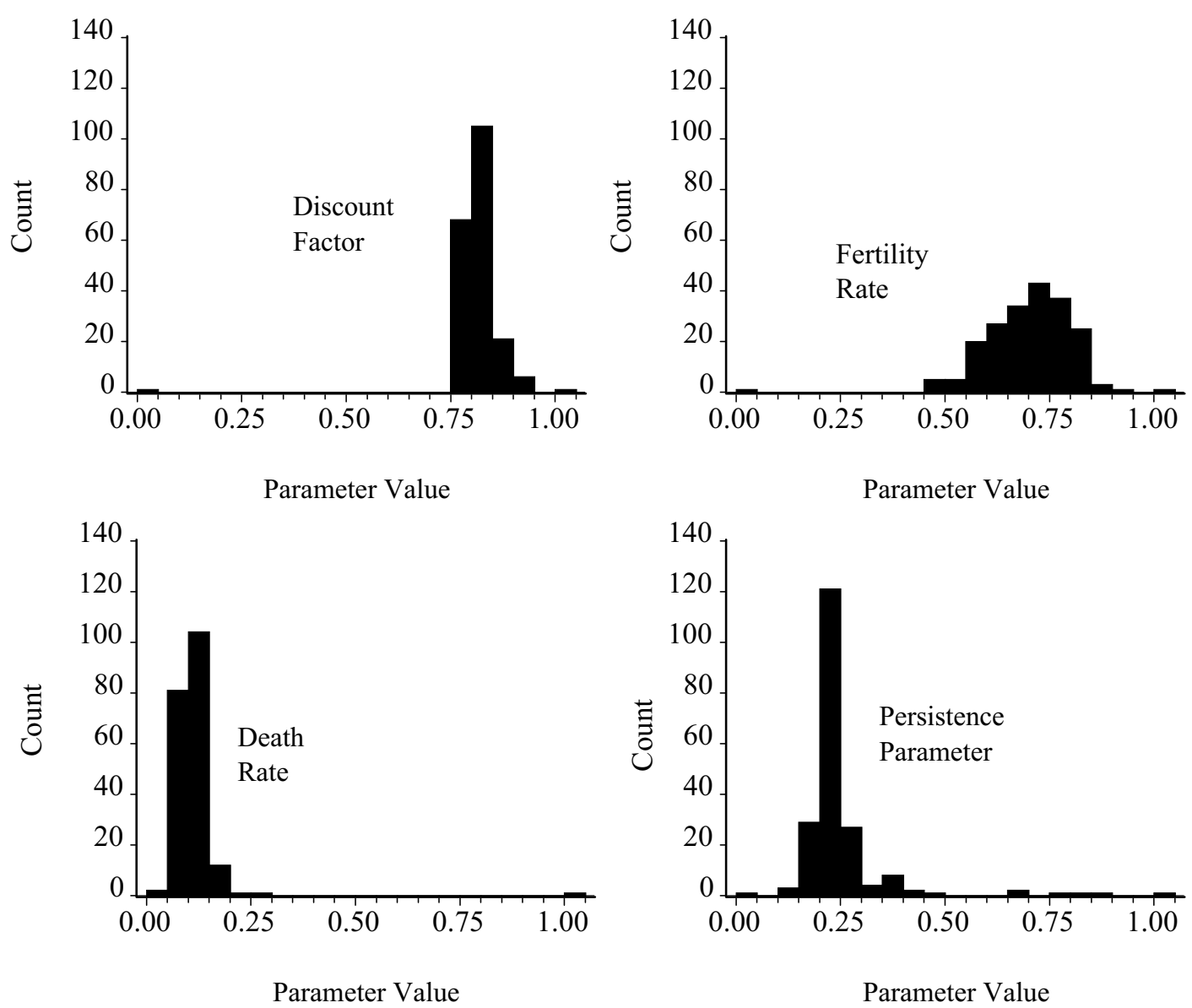

Notes to Figure: Estimated sampling distributions are based on 200 bootstrap replications. 


\section{Figure 6}

Model Spectra, and Data Spectra Confidence Tunnels

\section{U.S. Cattle Consumption and Stock}
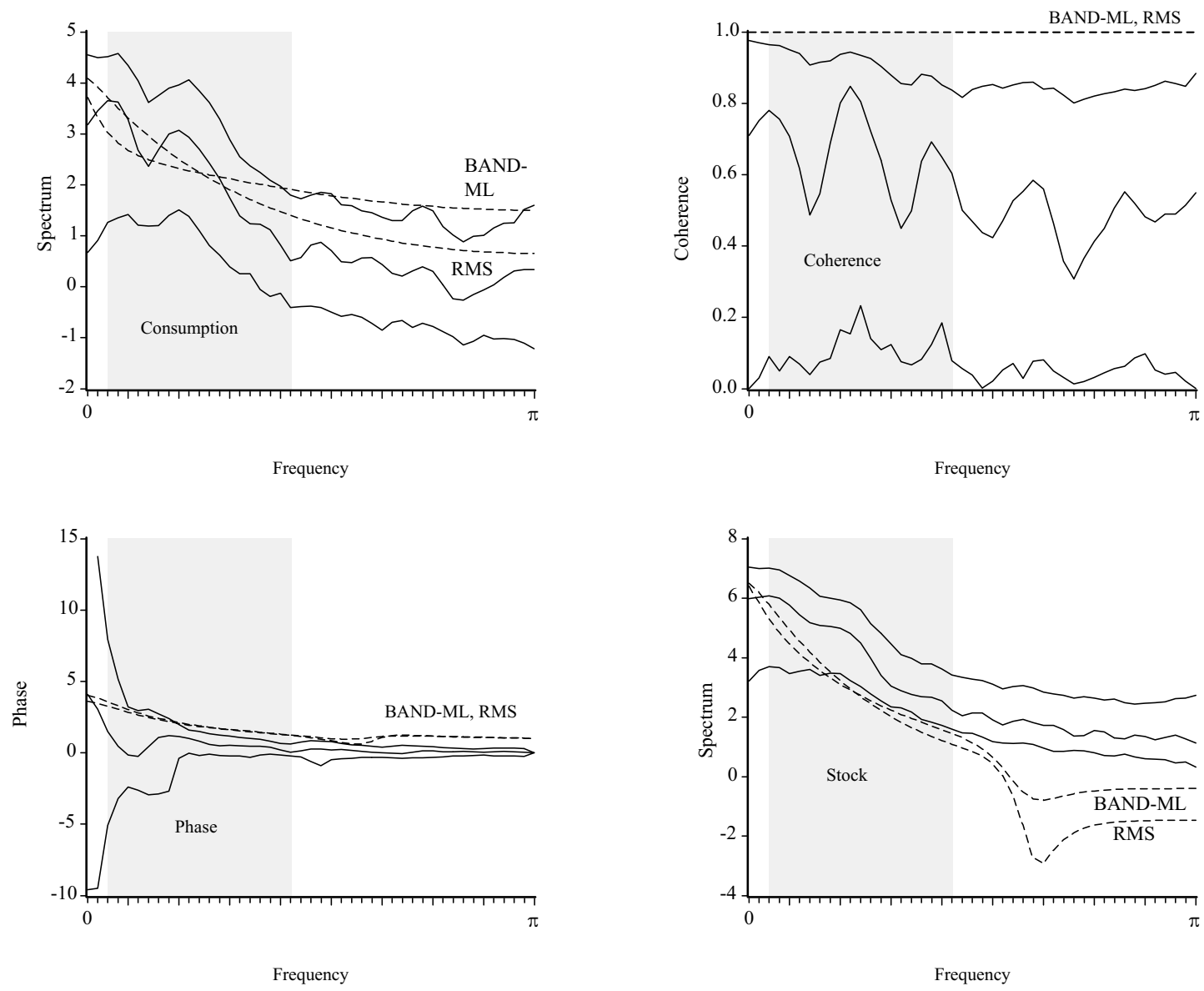

Notes to Figure: We show the $90 \%$ confidence tunnel for the data spectrum, together with the model spectrum evaluated at the band-restricted maximum likelihood parameter values, for each element of the spectral density matrix. The shaded frequency band corresponds to cycles with periods of 30 to 4 years. 